DHPARTAMENTO DE ANATOMIA P'STOLGGICA

Dirctur: Prof. Dr. Juclydes Onofre Martins

\title{
RINOSPORIDIOSE EM MUAR
}

\author{
RHINOSIORIDIOSIS IN A MULI:
}

\author{
Antonio G. Fermi \\ JaYme Gavĩo Nítis \\ Assistente \\ Assistente \\ 2 estampas ( 3 gravuras)
}

Depois que Seeber (1900) descreveu a rinosporidiose como nova entidade nosológica, inumeras observações foram rẹistradas na literatura médica. Fm uma revisão bastante minuciosa da literatur3, Mello (1946) constatou terem sido descritos até aquela data cêrca de 4.27 caso.s em medicina humana e $37 \mathrm{~cm}$ medicina vcterinária.

l'osteriormente, o mesmo autor (1949), em outro trabalho, no qual fêz referência a novo caso em eqüino, aumentou aquelas cifras para 442 e 39 , respectivamente.

Ao rever o assunto, constátamos outros trabalhos que nāo tinham sido considerados por aquêle autor. Assim Habini (19.17) estudou mais três casos de rinosporidiose conjuntival, Murga e Latibnida (1947) e Niño, Serrano e Pietro 119.19) relataram novas observaçōes na Argentina.

No Brasil, segundo ALMEjda (1939), o primeiro caso foi encontrado em Sião Paulo cm 19:30 por Montenegro, que não publicou sua observação. Seguiram-se os trabalhos de Findino e col. (1935), Barros Coklno (19:12), AbenAthar (1941) e Capua (1944).

Fim sua tese de professorado, Fialmo (1916) fêz referência à nova observação pessoal, ben como cita o caso estudado por MacClure.

Frikeira Fihro e Monteino Saldes (1919) relataram novo caso em paciente que havia residido na India e Souza C CrRruti (1951) apresentaram um taso de rinosporidiose ocular, ao VII Coneresso Brasileiro de Oftalmologia.

Lacaz (1953) em seu livro reproduziu a microfolografia da observaçáo de Soura Dras, a qual não foi publicada.

Podemos, pois, estimar em 450 o número de observações desta doença, na literatura mundial, das quais apenas 10 foram verificadas no lirasil.

As observaçōes em Inedicina veterinåri̊ se acham resumidas na Tabela $f$, organizada por Mro.to em sua lese para concurso de cáledra e enriquecida com 
'IABEI,A I

(ASOS DI: RINOSIPUIIOSE ANIMAI, OBSFRVAIOS FM TOI) M(NI)(*)

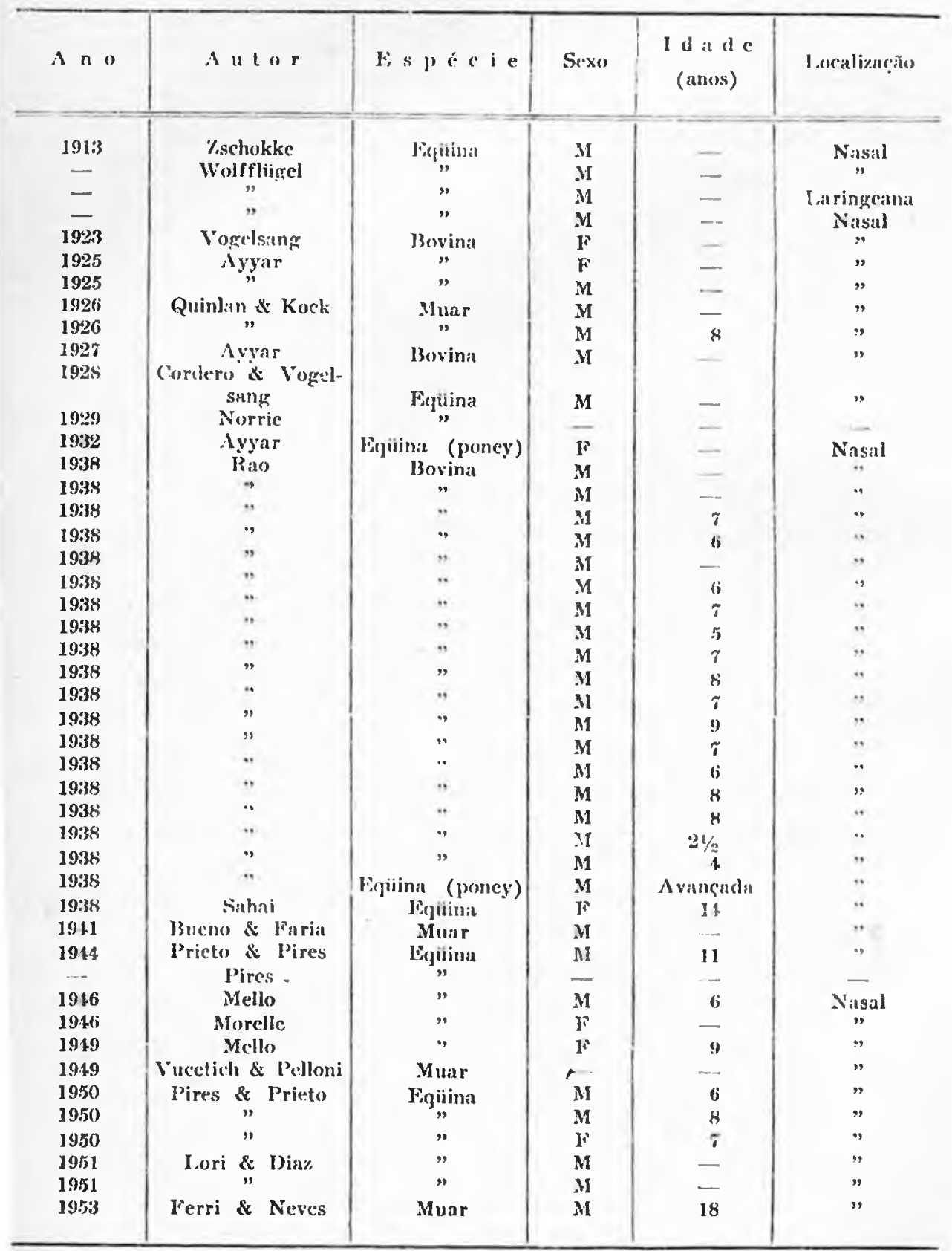

(*) Tabelit organizada por Mers.o (19k6), com pe(jurnas inodificações. 
os caso: pullicados por Vlcetich e Peis.osi (1949), os quais estudaram nova incidencia em muar, por Pirks e Prieto (1950), gue relataram : casos em crfïnos c por Lokt e Disz (1951), que assinalaram mais 2 casos em eqüinos.

Via literatura veterinaria nacional, a primeira referencia a esta micose foi filla por Bt fano e Faria (1911), estudando um caso em muar procedente da

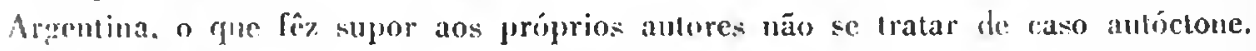
Siguiram-se os trabalhos de MLitro registrando um caso em P.S.I. e de Morelt.k. 11916) que relala nova obscrvação em fega meio sangue inglés. ambes no liio (irande do sul.

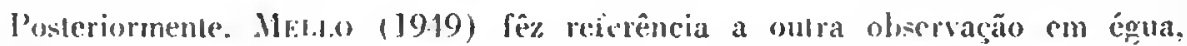

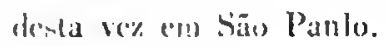

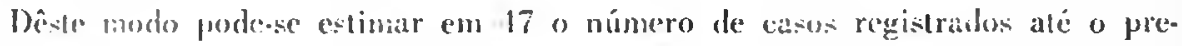

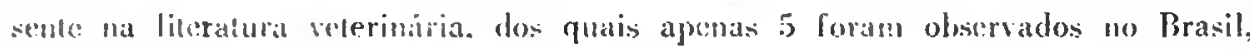
inchuindo a prexirile obsersação.

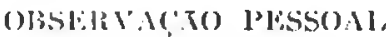

() material procedente de Sorocala (Estado de São Paulo) foi retirado cirurgicanente do vesclibulo nasal direito de sm muar do sexo masculino, com li? anos de idade, em 30 de setembro de 1952, pelo Dr. J. Bela Veto.

Srgundo informasão do proprielírio, a neoformaçäo estava evoluindo há 1 ano, prrojudicara a respiraçäo do animal o sangrava freqüentementr.

la messia localidade existiam outros animais que. porém, nada apresenlavam.

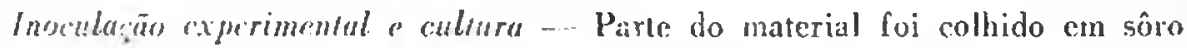
fisioligyico e foram inoculados 2 ralos. 2 cochos e 2 cães, no saco conjuntival c no tecidn celular subcutâneo. Também foi inoculado um cqüino, na fossa nas:al, for escarificaçāo. Os animais foram necropsiados unra período variável de 2 at 12 meses e os cortes histológicos cios pontos de inoculação nada revelaran.

Parte do material colhido em sôro fisiológicon foi enviado ao Departamento de Microhiologria e Imunologia da Faculdade de Medicina Veterinária da Universirlade de São Paulo, onde foram tentadas culturas semeando-se o material em diversos meios. Tódas as tentativas deram resultados negativos.

fixame ammomopatoligico - A peȩa por nús recebida era um polipo pe-

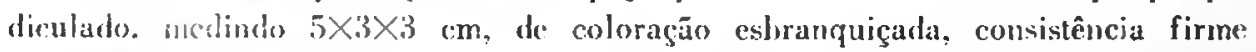
com risuras de $1 \mathrm{~cm}$ de profundidade, e de superficic muriforme, isto 6 fina. mente granulosa. 
0 material foi fixado em formol neutro e fisiológico a $10 \%$. Alguns fragmentos foram cortados em micrótomo de congelação e corados pelo Fiscarlate R. Outros fragmentos foram incluídos em parafina e corados pelos seguintes métodos: hematoxilina e eosina, Bielshowsky, hematoxilina férrica de Heidenhain e reação de Hotchkiss.

Exame hislopalológico - Verificou-se ato microscópio que o polipo se mostrava revestido externamente parte por um epitćlio prismático estratificado, e parte por epitélio pavimentoso estratificado ligciramente queratinizado. Em alguns pontos êste epitélio se apresentava ulcerado e recoberto por membrana fibrino-purulenta. No córion encontrou-se alguns brotos epiteliais, muitas vêzes com parasitas no seu interior, bem como alguns ductos glandulares que em certos casos apresentavam também parasitas no seu interior.

Logo abaixo do epitélio encontrou-se um tecido granulomatoso rico em vasos neoformados, muitas vêzes com células endnteliais salientes. Os capilares mostravam-se em geral dilatados e cheios de sangue. Verificou-se ainda uma intensa proliferação de fibroblastos e células histiocitárias. Ao redor de algumas formações parasitárias havia uma verdadeira cúpsula constituída por fibrócitos. En. tre êstes elementos encontrava-se intensa infiltraçāo leucocitária onde predominavam os polimorfos nucleares neutrófilos, principalmente $\mathrm{cm}$ certos campos. Em outros predominavam os linfócitos e plasmócitos. Os polimorfonucleares eosinó. filos foram encontrados em muitos campos, porém não em grande número. Nas proximidades de algumas formas parasitárias foran encontrados gigantícitos "tipo corpo estranho". Algumas zonas do polipo se apresentavam com hemorragias e com células do S.R.F. carregadas de pigmento hemossiderótico. Nos cortes im. pregnados pela prata (método de Bielshowsky") verificou-se que o tecido reticular se apresentava hiperplasiado, formando una rềde ou cî́n em tôno dos pa. rasitas.

Na massa do tecido granulomatoso bem como englobados no epitélio como acima foi referido, verificou-se a presença de formas arredondadas ou ovais do parasita em diferentes estádios de evoluçäo. lissas formas podem ser divididas em duas classes distintas, como aliás faz a rnaioria dos auiore's.

1. Forma joiem. Nesta forma os parasitas se apresentavam como elementos circulares ou ovalados, limitados por espêssa membrana fortemente cosinofílica c contendo no centro um núcleo. À inedida que estas formas vão crescen. do, o citoplasma se carrega de gotículas de gordura, que foram evidenciadas pelo escarlate $R$, e nos preparados corados pela hematoxilina e cosina apresentava-se com aspecto esponjoso.

Iim muitas destas formas só foi possícl dislinguir a mecahrana envolvendo un grande sacúolo. 
2. Forma adulia. Os parasitas, à medida que o núcleo vai se multipli. cando, passam da forma jovem à adulta e como tal se apresentavam como elementos grandes $(300 \mu)$, arredondados, limilados por uma membrana e contendo no interior numerosos esporos de tamanhos variados (até $9 \mu$ segundo Mri.to), is quais possuiam um cariosoma e cram elivoltos por uma membrana.

O parasita em sua forma adulta é repressentado por um esporângio ou ascocarpo.

Alguns dos esporûngios se apresentavam em nosso material com a membra. na rôta, dando saida a esporos, outros se mostravam sem esporos e cheios de lcucócitos e outros ainda eram representados apenas jelas membranas, as quais estavam enrugadas e rodeadas por células do infiltrado inflamatório, entre as quais existiam numerosos esporos.

Deve-se dizer, finalmente, que nos preparados em que foi feita a rcação de llotchkiss, tanto as membranas das formas jovens, como as dos esporângios e as dos esporos, deram resultados positivos.

\section{Discussau}

Inümeros problemas têm sido levantado: em relação à rinosporidiose pelos diferentes pesquisadores que se têm preocupado com esta doença. Uns estão aparentemente resolvidos, outros aguardam soluçăo. Verifica-se na literatura que - parasita foi considerado por SEeBer, O'OKIyealy (1903) e por outros que og seguiram como um protozoário e posteriormente foi por AsHworth (1923) co. locado no reino vegetal entre os Phycomycetes na ordem Chytridiales. Dai por diante a maioria dos micologistas vem aceitando esta classificação. ZscrıoKKe. (1913), ao descrever pela primeira vez a rinosporidiose em equiino, procurou criar nova espécie, o $R$. equi; para o parasito dos bovinos, Allen e Dive (1936) tentaram criar outra espécie $R$. ayyari, por ter sido descrito por AyYals (1925), e posteriormente ABris-AtHar também tentou criar nova espécie, o $R$. amazonicum, colocando-o entre os Ascomycetes. Os autores que estudaram o assunto comparativamente não encontraram razões para a criação de novas espécies, ponto de vista que subscrevemos, pois, comparando a morfologia do parasita do presente caso, com a de material humano gentilmente cedido por lacaz e Cerruti, não encontrímos diferenças. E’ de se salientar que CakiNi (1940), ao descrever formas parasitárias cm cistos da pele de rãs, álendendo ao tamanho dos parasitas e ì distância na escala zoológica do hospedador, preferiu, até novas verificaçōes, criar o gênero Dermosporidium, com a espécie $D$. hylarum, cmbora a morfologia dèstes parasitas fôsse idêntica à daqueles que ocorrem nos animais superiores. 
Esta nicose tem sido constatada nuilo mais freqü̈entemente c'm medieina humana do que entre os animais, o que talve ocorra pela dificuldade de diagniotico em medicina veterinária.

Fintre os animais tem sido descrita simente en equinnos, inuares e hovino.

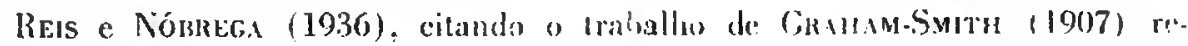
gistraram casos de rinosporidiose em psitacídeos, porém, a consulla do trabalho original daquele autor não autoriza a concluir que se trate da mesma docnça e o próprio autor não chegou ì conclusão defunitiva - "Though differing in many respects this parasite more closely resembles Rhinosporidium kinealyi than any other cyst producing protozoon" (loc. cit., pg. 556).

lintre os animais tem sido verificada a doença na seguinte proporşão:

$$
\begin{aligned}
& \text { Bovinos } \ldots \ldots \ldots \ldots \ldots \ldots \ldots .22 \text { casos }(13,4 \%) \\
& \text { Equiinos } \ldots \ldots \ldots \ldots \ldots \ldots \ldots . \quad 19 \text { casos }(41,3 \%) \\
& \text { Muares } \ldots . . \ldots \ldots \ldots \ldots \ldots \text {. } 5 \text { casos }(10.9 \%)
\end{aligned}
$$

Este granuloma tem sido descrito etn diferentes raças, não parecendo t'r qualquer especificidade, como já havia sido assinalado por Mr.t.o. ontre outro:.

A incidência maior em indivíduos do sexo masculino. tanto em medicina humana como em veterinária é um problema, até hoje, sem explicação.

Em relação à idade, a consulta da Tałela l mostra que os animais são afetados com maior frequiência entre 6 e: 8 anos de idade. com os limites extremo: de $2 \frac{1}{2}$ e 18 anos.

() polipos rinosporidiásicos sillam-se cm geral nas fossas nasais, mas - cilam-se casos com localização conjuntival (Kuntsakatne, 1936, HabiBi, 1917,

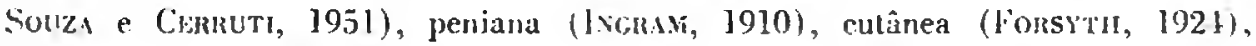
generalizada (ALLev e Duve, 1936) e cercbral (Arsssivinks, 1926), Cormas

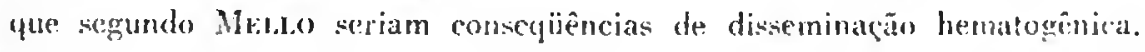

Entre os animais, a localização é de quase $100 \%$ ć na fossa nasal, exceção foita à ohservação de Workrü̈rce. (1913), na qual os polipos situavam-se no laringe.

Outro problema que tenr preocupado us estudiosos é o modo de infeç̧̄on. KarLnaratse. (1936) e Rao (1938) sugericam a possibilidade da infecção alràvés da água ou da pocira. A incógnita permanecerá provivelmente até que séja conseçuida a cultura do parasila e a infecģio experimental. "que tem sido ten. tado por inúmeros autores, entre os guais Rao, Bulno e Fikla, Mello e tamhém por nós, mas sempre com resultados ncgativos. 
Quimto i lesão. o mesmo lipo de tecido granulomatoso descrito por todos os pesquisadores foi lambém observado em rosso material. Alguns autores, enIre os quais Tito e Nosro (1912) tem chamado a aten(ĩo) para uma hiperplasia (i) tecido reticular, curolvendo o parasita cono um cèsto, fato que tambím ol. servímos uns preparados corados pelos métodos argênticos.

li: de se salicutar, fintmente, que as membranas dos esporângion e los es. poros deram reagin positiva pela lécrnica de Hotchisiss, cridenciando, pois, sua balureza polissacarídica.

\section{SLHARIO J; coNelisolis}

Depus da revisian da literatura. en que á apresentadia uma labela resumindo os cuso publicados na litcratura universal, é descrito um caso de rinosporidiose an: muar. Descrescese macro e microscopicamente o polipo, confirmandose a descricáa de outros pespluisadores, bom comm a hiperpiasia do retículo ao redor dos parisitas. Demonstrase a naturera polissacarídica das membranas dos es-

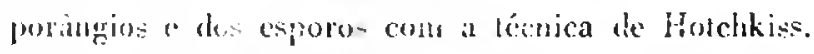

As tentuivas de cultura $\mathrm{cm}$ discrsos sneios e a inoculação experimental cm varios animais foram negativas. Os dados sĩo discutidos à luz da literalurá, bem coino us fatores de incidencia da doenga, relativos is especie, raça. seso e idade.

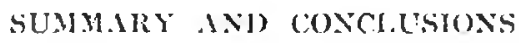

Afer review of the literabure. includine a list of cases already published. a case of thinosporidiosis in a mule is reporied. Macro and microscopic descrip). lions of the polyp ire made, corroborating those given by other auhors as well an the reticulum hyperplasia around the parasites.

The polysaccharide nature of the membranes of sporangia and spores was demonstrated through Hotchkiss's method. Attempts to cultivate the fungus in different mediums, as well as experimental innoculation in various animals. had negatior results. Besides these evidences, the factors of incidence of the disease, as relatid to species, breed, sex and age, are discused comparatively with the literalure.

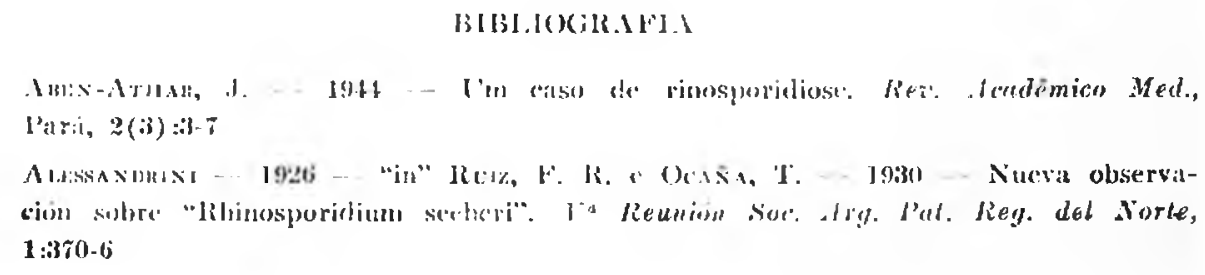


Alufe, D. R. W. K. e Dave, M. L. - 1936 - “in" Mri.o, M. 'l". - 1946

Armaida, F. - 1939 - Mycologin Médica: 412-50, Sĩo 1'aulo, ( $i_{i}$. Melhorimentos Asitworti, J. IH. - 1923 - “in" M\&t, M, M. T. - 1916

AYYAB, V. K. - 1925 - "in" AYYAR, V. K. - 1932 - Rhinosporidiosis in "(puines. Indinn Jour. Vot. Sci. \& Anim. Husbandry, 2:49-52

Barmos Conbro - 1942 - I'm caso de rinosporidiose nasil. Resenha Clin. Ciene. Inst. Lorenzini, Sỉo P’aulo, 11:521

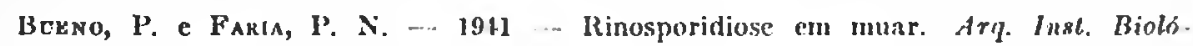
gico, Săo Puulo, 12:297-:302

Capua, M. - 1944 - Consideragóes em tôrno de seis observaçós de polipo sangrante do septo. An. M'sulixt. Med. Cirur, 47(4):351

Carist, A. - 1940 - Sübre um parasito semelhante ao "IRhinosporidium" cacon. trado em quistos da pele de uma "Hyla". Arq. Inst. Biológico, São Paulo, 11:03-6

Fegheika Fu.ho, M. A. e Mosizino Saliks, F. J. - 1949 - Polipo do sejpto nasal pelo "Rhinosporidium sceheri" - "in" LAc.ar, C. S., 1953

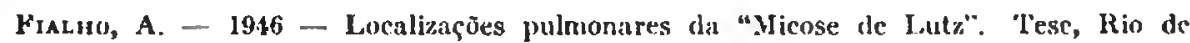
Janeiro

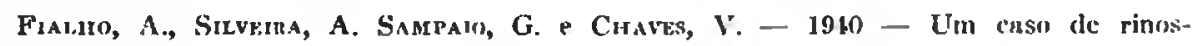
poridlose nasal (nota prévia). IInspital, lRio de Janeiro, 17(6):94,5-(i

Forsytr, W. I. - 1924 - "in" Mre.to, M. T. - 1916

ínнма-Sмгті, G. S. - 190\% - A cystic disease of the heart, gizzard and nusches of young grass parakects (Psittic:us undulatus) due to a protozoon parasite. Jour. IIyg., $7: 552-7$

HAlıu, M. - 1947 - "Rhinusporidiuu secberi" en Iran. Aun. Purnuit. IIumasine et Comp., $22: 84-8$

lNolis, A. C. - 1910 - “in" Me1.10, M. 'T, 1946

Kakvsaratxi, W. A. F. - 1986 - The pathology of Rhinosporidiosis. Jiner. Path. \& Bact., $42: 193-202$

Lacaz, C. S. - 195i - Manunl de Micologia Midicat 261-r̃z, Săa Paulo, Pont. Univ. Catúlicin

1,он, R. С IM\%, 13. C. - 1951 - l'oliposis nasil en equinos por "Rhinuspuridiur" sceberi". Gac. Vet., Bs. Aircs, 18(71):131-5

Mer.o., M. 'T. - 1916 - listudus sobre "Rhinosporiclinm seceberi". 'l'ese, Rio de" Janeiro

Mraro, M. 'T. - 1999- Rhinuspuridiosis. Mycopathologia, $4(1): 3128$

Moura, (). - 1946 - Rhinosporidlose coüina no Rio Girande do sul. Mol. Mir. Jrod. Animal, Pïrio Alegre, $2(4): 3.5-9$

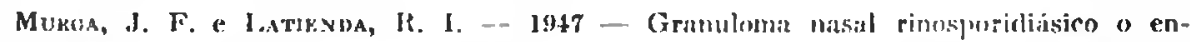

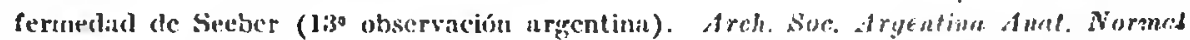
y l'atol., 9(1):113-9 … “in" Biol. Absl., 19;0, 24: 15is (Ret. 19.86\%) 
Nìno, F. SEuraxo, B3., Dr P'IEtro, A. - 1949 - Granuloma rinosporicliásico o enfermidad de Sceber por "Rhinosporidium Secheri" (11* obstrvación argentina). Jez. sis. Med. Argent., 63:114-6

Whinki.Y, F. - “in" Mriro, M. T. - 1916

P’nes, A. e l'nuro, C. - 1950 - Tres nuevos casos de rinosporidiosis en cabnllos. Uac. Vat., J3s. Aires, $12(65): 118-25$

Lian, A. N. M. -. 1938 - Rhinosporidiosis in bovines in the Madras Presidency, with is discussion on the probiable modes of infection. Indian Jour. Vet. Sci. $\&$ Anim. Huslindry, 8:18i-9:

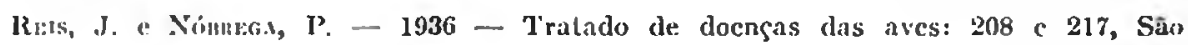
Piaulo, Cia. Melhuramentos

Strima, (;. R. - 1900 - Un nnevo esporozoario parasito del hombre. Dos casus encontridos en polipos nassiles - "in" Alsmima, I". - 1939

Sovz; I. I. - Crmut, II. - 19 ij - Um caso de rinosporidiose ocular. Apres:ntado an Vllo Congresso brasileiro de Oftalmologia, de 11 a 17 de jullo, no Licio de Janciro

"Аato e Mosto - 1912 - "in" Mello, M. T - 1916

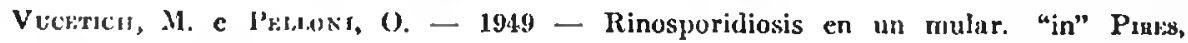
A. e PRiero, C. -1950

Worlflïgi: - 1913 - "in" Voglisang, E. G. - 1923 - "Rhinosporidium Seeberi", parasito del bovino - $117^{\circ}$ Conf. Sud. Amer. de IIig. Microb. y l'atol., 2:305

ZschoKke, X. - 1913 - “in" AlMeinA, F. - 1939 



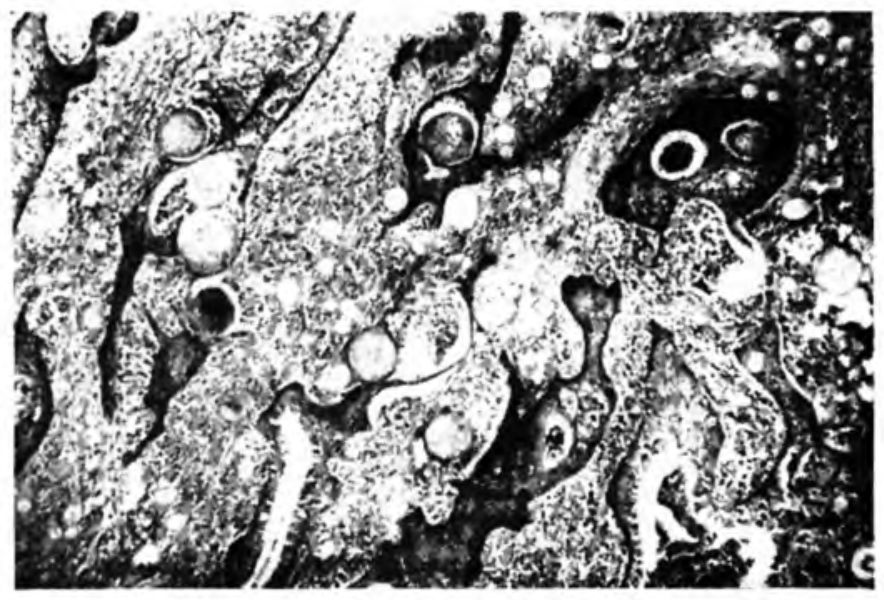

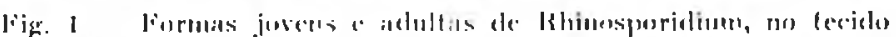

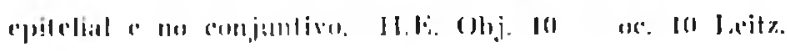

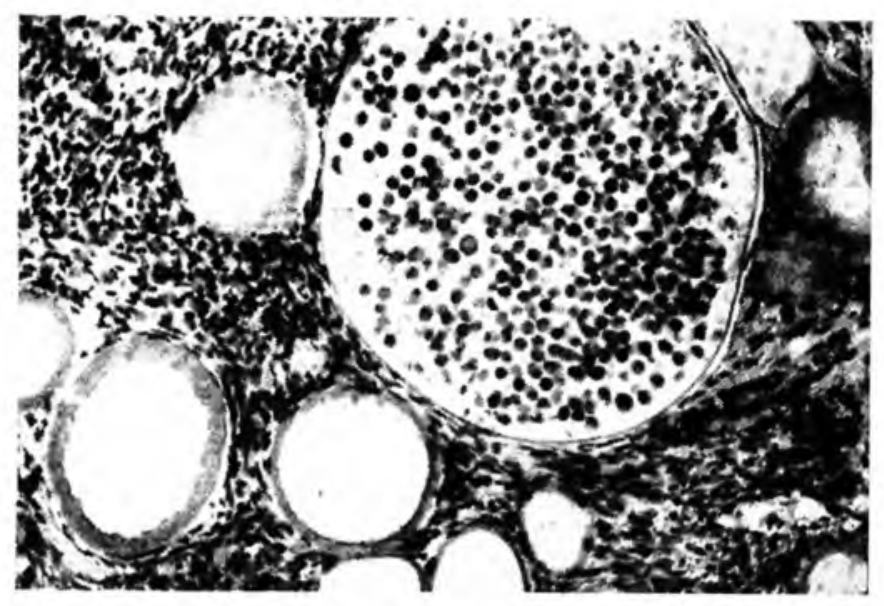

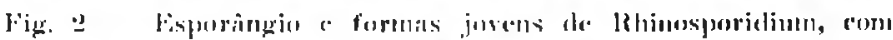

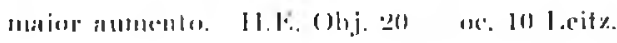




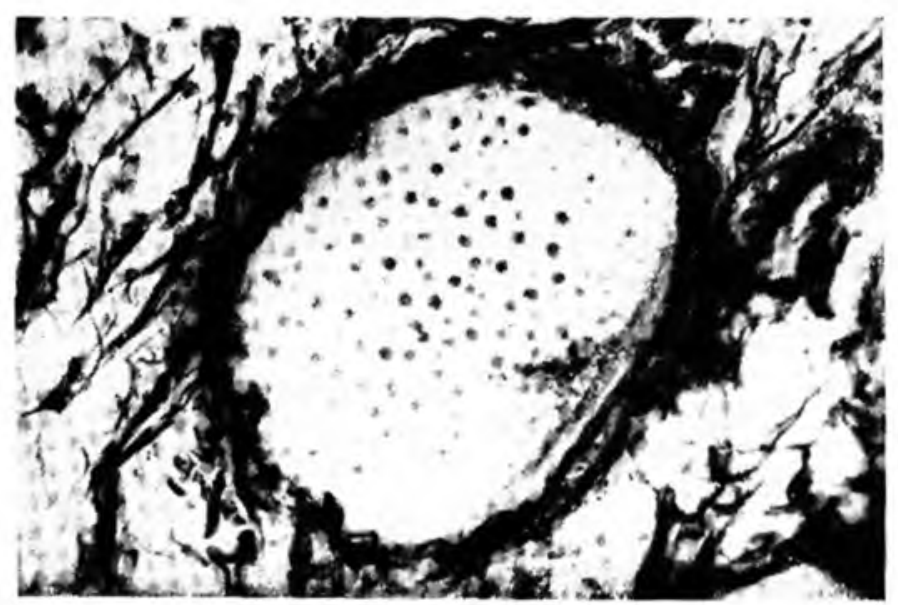

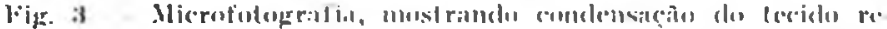

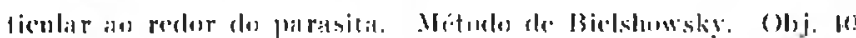
ac. In lieil\%. 